\title{
Functional properties of DENV EDIII-reactive antibodies in human DENV-1-infected sera and rabbit antiserum to EDIII
}

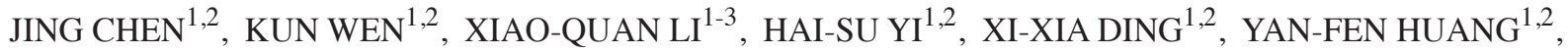

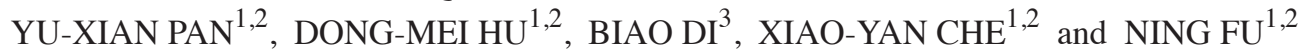 \\ ${ }^{1}$ Laboratory of Emerging Infectious Diseases; ${ }^{2}$ Division of Laboratory Medicine, Zhujiang Hospital, \\ Southern Medical University, Guangzhou, Guangdong 510282; ${ }^{3}$ Guangzhou Center for Disease Control and Prevention, \\ Guangzhou, Guangdong 510440, P.R. China
}

Received April 21, 2015; Accepted March 14, 2016

DOI: $10.3892 / \mathrm{mmr} .2016 .5454$

\begin{abstract}
The envelope domain III (EDIII) of the dengue virus (DENV) has been confirmed to be involved in receptor binding. It is the target of specific neutralizing antibodies, and is considered to be a promising subunit dengue vaccine candidate. However, several recent studies have shown that anti-EDIII antibodies contribute little to the neutralizing or enhancing ability of human DENV-infected serum. The present study involved an analysis of the neutralization and antibody-dependent enhancement (ADE) activities of EDIII-reactive antibodies in human convalescent sera from patients with primary DENV-1 infection and rabbit antiserum immunized with recombinant DENV-1 EDIII protein. The results indicated that serum neutralization was not associated with titres of EDIII-binding antibodies in the human DENV-1-infected sera. The depletion of anti-EDIII antibodies from these serum samples revealed that the anti-EDIII antibodies of the patients contributed little to neutralization and ADE. However, the EDIII-reactive antibodies from the rabbit antiserum exhibited protective abilities of neutralization at a high dilution $(\sim 1: 50,000)$ and ADE at a low dilution $(\sim 1: 5,000)$ for the homotypic DENV infection. Notably, the rabbit antiserum displayed ADE activity only at a dilution of 1:40 for the heterotypic virus infection, which suggests that EDIII-reactive antibodies may be safe in secondary infection with heterotypic viruses. These results suggest that DENV EDIII is not the predominant antigen of the DENV infection process; however, purified or recombinant DENV EDIII may be used as a subunit vaccine to provoke an effective and safe antibody response.
\end{abstract}

Correspondence to: Professor Ning Fu, Laboratory of Emerging Infectious Diseases, Zhujiang Hospital, Southern Medical University, 253 Gong ye da dao zhong, Guangzhou, Guangdong 510282, P.R. China

E-mail: zfzn2013@126.com

Key words: envelope domain III-reactive antibodies, neutralization, antibody-dependent enhancement, human dengue virus-infected sera, rabbit antiserum

\section{Introduction}

Dengue is a mosquito-borne viral disease caused by four serologically and genetically related viruses termed DENV-1, DENV-2, DENV-3 and DENV-4. Infection with each DENV serotype causes an array of clinical diseases, ranging from dengue without warning signs, dengue with warning signs to severe dengue-dengue haemorrhagic fever and dengue shock syndrome (1-4). Individuals infected with one serotype can acquire lifelong homotypic immunity (5); however, those suffering secondary DENV infection with another serotype may have a greater risk of progressing to severe dengue (6). Antibody-dependent enhancement (ADE) by pre-existing cross-reactive, weakly neutralizing antibodies has been suggested to be important in disease severity by promoting virus entry into Fc $\gamma$ receptor-bearing cells (7). The presence of ADE impedes the development of dengue vaccines to induce protective neutralizing antibodies without enhancing viral replication.

The surface of the mature DENV particle is covered with 90 head-to-tail homodimers of envelope glycoprotein (E) (8), which is the major surface protein of flaviviruses involved in multiple processes, such as viral adsorption, membrane fusion and cell tropism. The $\mathrm{E}$ protein ectodomain can be divided into three distinct structural domains designated as I (EDI), II (EDII) and III (EDIII). Structurally, EDIII has been proposed to be a putative receptor-binding domain (9). EDIII-reactive antibodies are found in convalescent sera from patients infected with dengue $(10,11)$.

The EDIII-reactive antibodies can function as effective neutralizers and also may possess varying degrees of enhancing activity (12-19). Recent studies have revealed that these antibodies from virus-infected serum are considered to contribute little to neutralizing and enhancing activities (11,20-22). Thus, it could be presumed that antibodies from patient sera are induced by a number of different epitopes on complex DENV in vivo, and not all protective epitopes can serve as predominant epitopes to provoke an effective neutralizing antibody response or ADE; however, protective non-predominant epitopes may serve as purified subunit vaccine candidates to induce a protective response. Additionally, the majority of research on the role of EDIII-reactive 
antibodies of human convalescent serum focused on DENV-2 and DENV-3 infections (11,22), however, $>60 \%$ of patients with dengue in southern China have DENV-1 infection. In this study, therefore, neutralization and ADE activity as well as the EDIII-binding antibody titre on homotypic and heterotypic DENVs in human DENV-1-infected sera and rabbit antiserum immunized with recombinant EDIII protein were evaluated. Furthermore, the contribution of EDIII-reactive antibodies was verified by depleting EDIII-reactive antibodies from human sera and rabbit antiserum using Dynabead-EDIII conjugates.

\section{Materials and methods}

Ethics statement. The current study was approved by the ethics committee of the Zhujiang Hospital of Southern Medical University (Guangzhou, China).

Patients. The DENV-1-infected convalescent patients were recruited by follow-up phone calls and achieving consensus. A total of 30 primary DENV-1-infected convalescent human serum samples were collected from the Guangzhou Centre for Disease Control and Prevention (Guangzhou, China). The convalescent human serum samples were obtained by venous blood.

Animals. New Zealand white rabbits $(n=3)$ were purchased from the Laboratory Animal Centre of Southern Medical University. The animals were housed at $25^{\circ} \mathrm{C}$, with $60 \%$ relative humidity and free access to food and water. The serum was collected from the jugular vein of the rabbit after anesthesia. The rabbits were sacrificed with pentobarbital $(30 \mathrm{mg} / \mathrm{kg}$ weight) at 9 weeks.

Cells and viruses. LLC-MK2 (\#CCL-7) and K562 (\#CCL-243) (American Type Culture Collection, Manassas, VA, USA) cells were cultured in a $5 \% \mathrm{CO}_{2}$ incubator at $37^{\circ} \mathrm{C}$ supplemented with $10 \%$ fetal bovine serum (FBS, Gibco; Thermo Fisher Scientific, Inc., Carlsbad, CA, USA) in minimal essential medium (MEM; Gibco, Thermo Fisher Scientific, Inc.) and RPMI1640 medium (Gibco), respectively. The four dengue serotype strains (DENV-1, Hawaii; DENV-2, New Guinea-C; DENV-3, Guanxi-80-2; and DENV-4, H241, provided by the Center for Disease Control and Prevention of Guangzhou, China) used in this study were propagated in C6/36 Aedes albopictus cells American Type Culture Collection; \#CRL-1660) in MEM containing $10 \% \mathrm{FBS}$ at $33^{\circ} \mathrm{C}$ for three to five days in the presence of $5 \% \mathrm{CO}_{2}$ as described previously (23).

Expression, purification and labelling of DENV recombinant EDIII proteins. DENV rEDIII proteins of the four dengue serotypes were produced as described previously (24). In brief, the genes encoding DENV EDIII of the four DENV serotypes were amplified and cloned separately into a pPIC9K vector (Invitrogen, Thermo Fisher Scientific, Inc.), and the 6X His tag coding sequence (synthesized by Sangon Biotech Co., Ltd., Shanghai, China) was added downstream. Recombinant plasmids were then transferred into Pichia pastoris GS115 (Invitrogen, Thermo Fisher Scientific, Inc.). The expression of rEDIII protein for each DENV serotype was initiated using methanol induction, and the protein was subsequently purified using Ni-nitrilotriacetic acid affinity chromatography (Qiagen, Hilden, Germany). The purified rEDIII protein was used to prepare the Dynabead-histidine-tagged rEDIII conjugates (Thermo Fisher Scientific, Inc.) and horseradish peroxidase (HRP)-rEDIII conjugates by a periodate method (25).

Serum samples. A total of 30 primary DENV-1-infected convalescent human serum samples confirmed by RT-PCR and a dengue IgG and IgM capture enzyme-linked immunosorbent assay (ELISA; Panbio, Windsor, Australia) were collected from the Guangzhou Centre for Disease Control and Prevention (Guangzhou, China). The 30 serum samples were obtained from patients aged 10-75 years, 15 males and 15 females. The control sera were obtained from healthy individuals without dengue. The collection and use of human sera were in compliance with the Ethics Committee of the Zhujiang Hospital of Southern Medical University (Guangzhou, China). Rabbit antisera against DENV-1 rEDIII were prepared in New Zealand white rabbits using the following procedure: The rabbits were subcutaneously immunized with $500 \mu \mathrm{g}$ purified DENV-1 rEDIII emulsified with Freund's adjuvant (Sigma-Aldrich, St. Louis, MO, USA) at ten-day intervals for 8 weeks followed by intravenous boosters at three days prior to serum collection. purified DENV-1 rEDIII $(50 \mu \mathrm{g})$ was injected through the ear marginal intravenously. Three days later, the serum was collected from jugular vein of the rabbit after pentobarbital anaesthesia.

RT-PCR was performed according to a previously described protocol (26). Briefly, RT-PCR was performed using an ABI 7300 PCR thermal cycler (Applied Biosystems; Thermo Fisher Scientific, Inc.). With the DEN-1 assay as an example, the PCR mixture included a final volume of $20 \mathrm{ml}$ with $10 \mathrm{ml}$ one-step RT-PCR Master kit (Qiagen China Co., Ltd., Shanghai, China), $0.5 \mu \mathrm{l}(10 \mathrm{pmol} \mathrm{ml} / \mathrm{l})$ each primer, $1.5 \mu 1(2 \mathrm{pmol} \mathrm{ml} / 1)$ probe, $5.5 \mu \mathrm{l}$ distilled water and $2 \mu \mathrm{l}$ extracted RNA. The amplification conditions were as follows: An initial step at $50^{\circ} \mathrm{C}$ for $30 \mathrm{~min}$ and $95^{\circ} \mathrm{C}$ for $15 \mathrm{~min}$, followed by 40 cycles of $95^{\circ} \mathrm{C}$ for $15 \mathrm{sec}$, annealing and elongation at $60^{\circ} \mathrm{C}$ for $60 \mathrm{sec}$, with fluorescence acquisition in single mode. Negative controls included human RNA, C6/36 RNA, distilled sterile water and PCR mixture. All experiments were repeated four times. The PCR mixture for the remaining three DEN assays were the same as for DEN-1. Amplified products were detected by agarose gel electrophoresis and sequence identification. The sequencing was undertaken on an ABI PRISM 3730 DNA Sequencer. Primers and probes used in group-specific and serotype-specific real-time assays for DENV are as previously described (27).

Detection of EDIII-binding antibodies by double-antigen sandwich ELISA. A double-antigen sandwich ELISA was performed to evaluate the titres of the EDIII-binding antibodies in the serum samples described above. Microwell plates (Costar Corning, Inc., Corning, NY, USA) were coated with $100 \mu \mathrm{l} /$ well DENV rEDIII at a concentration of $0.1 \mu \mathrm{g} / \mathrm{ml}$ (for DENV-1 and DENV-2) or $0.05 \mu \mathrm{g} / \mathrm{ml}$ (for DENV-3 and DENV-4) overnight at $4^{\circ} \mathrm{C}$ followed by blocking with $2.5 \mathrm{~g}$ casein sodium salt, $1.21 \mathrm{~g}$ Tris-base, $2 \mathrm{~g}$ gelatin, $20 \mathrm{~g}$ sucrose, $0.2 \mathrm{~g}$ Merthiolate, and $5 \mathrm{ml}$ Tween 20 in

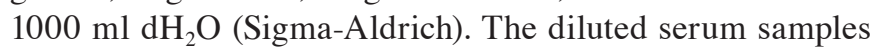
were added $(100 \mu \mathrm{l} /$ well $)$ and incubated for $1 \mathrm{~h}$ at $37^{\circ} \mathrm{C}$. After 
Table I. Neutralization titres and anti-DENV-1 EDIII serum titres of DENV-1-infected sera.

\begin{tabular}{|c|c|c|c|c|c|}
\hline \multirow[b]{2}{*}{ Patient number } & \multicolumn{4}{|c|}{ NT50 titres ${ }^{\mathrm{a}}$} & \multirow{2}{*}{$\begin{array}{l}\text { Anti-DENV-1 EDIII } \\
\text { serum titres }^{\mathrm{b}}\end{array}$} \\
\hline & DENV-1 & DENV-2 & DENV-3 & DENV-4 & \\
\hline 1 & 950.2 & 227.3 & 194.7 & 63.2 & 40 \\
\hline 2 & 1190.0 & 262.0 & 237.9 & 237.7 & 80 \\
\hline 3 & 1630.0 & 273.0 & 89.7 & 86.0 & 40 \\
\hline 4 & 1827.0 & 228.4 & 167.3 & 150.1 & 80 \\
\hline 5 & 1020.0 & 1023.0 & 918.5 & 271.6 & 40 \\
\hline 6 & 2896.0 & 2153.0 & 918.0 & 302.7 & 80 \\
\hline 7 & 2290.0 & 597.8 & 1155.0 & 276.2 & 40 \\
\hline 8 & 1316.0 & 2357.0 & 681.7 & 70.6 & 20 \\
\hline 9 & 1043.0 & 318.8 & 586.2 & 141.6 & 20 \\
\hline 10 & 587.1 & 108.1 & 164.6 & $<20$ & 5 \\
\hline 11 & 4114.0 & 1399.0 & 1061.0 & 368.1 & 80 \\
\hline 12 & 2666.0 & 302.2 & 159.4 & 543.7 & $<5$ \\
\hline 13 & 3708.0 & 133.1 & 36.2 & $<20$ & 10 \\
\hline 14 & 794.4 & 50.05 & 24.7 & $<20$ & 5 \\
\hline 15 & 529.7 & 103.4 & 331.2 & $<20$ & 10 \\
\hline 16 & 3666.0 & 141.8 & 204.2 & 48.9 & 20 \\
\hline 17 & 1419.0 & 164.9 & 142.0 & 102.5 & 20 \\
\hline 18 & 568.2 & 47.8 & 32.8 & $<20$ & 20 \\
\hline 19 & 1763.0 & 337.8 & 291.3 & $<20$ & 10 \\
\hline 20 & 2582.0 & 325.5 & 38.9 & $<20$ & 40 \\
\hline 21 & 1219.0 & 34.2 & $<20$ & $<20$ & $<5$ \\
\hline 22 & 1836.0 & 440.7 & 34.6 & $<20$ & 20 \\
\hline 23 & 153.6 & 28.35 & 28.5 & $<20$ & 20 \\
\hline 24 & 4707.0 & 630.6 & 1302.0 & 75.0 & 80 \\
\hline 25 & 962.5 & 88.4 & 129.3 & $<20$ & 10 \\
\hline 26 & 10684.0 & 742.7 & 2156.0 & 385.2 & 80 \\
\hline 27 & 240.4 & 68.8 & 79.0 & $<20$ & 40 \\
\hline 28 & 2013.0 & 395.2 & 75.7 & $<20$ & $<5$ \\
\hline 29 & 4960.0 & 634.5 & 503.0 & $<20$ & $<5$ \\
\hline 30 & 4178.0 & 57.6 & 44.3 & $<20$ & 20 \\
\hline
\end{tabular}

${ }^{a}$ NT50 neutralization titres of the sera are shown as reciprocal serum dilutions at which the viral infection was inhibited by $50 \%$. ${ }^{\mathrm{b}}$ Anti-DENV-1 EDIII serum titres are displayed the highest reciprocal dilution that gave an indicator of $>3$ standard deviations of the indicator produced for the control human sera. DENV, dengue virus; EDIII, envelope domain III; NT50, 50\% neutralizing titers.

washing, diluted horseradish peroxidase (HRP)-conjugated DENV rEDIII with $100 \mu \mathrm{l} /$ well was added to the plates, and an additional incubation was performed for $40 \mathrm{~min}$ at $37^{\circ} \mathrm{C}$. Finally, the colour reactions were visualized by adding tetramethylbenzidine (TMB, KPL, Gaithersburg, VA, USA) substrate $(100 \mu \mathrm{l} /$ well) and quenched with an equal volume of $0.3 \mathrm{~mol} / 1$ sulphuric acid. The absorbance was recorded at $450 \mathrm{~nm}$ on an ELISA plate reader (Bio-Tek, Winooski, VT, USA).

Depletion of DENV EDIII-binding antibodies from sera. Dynabeads (1- $\mu \mathrm{m}$ diameter) were conjugated with histidine-tagged rEDIII according to the manufacturer's protocol (Thermo Fisher Scientific, Inc.). Briefly, the Dynabeads were washed thoroughly 4 times with binding/wash buffer
(0.05 mol/1 sodium-phosphate, $0.3 \mathrm{~mol} / 1 \mathrm{NaCl}$ and $0.01 \%$ Tween-20) by standing the tube on a magnet for $2 \mathrm{~min}$ and discarding the supernatant. An excess of rEDIII protein was then added to the Dynabeads and mixed. The mixture was incubated on a roller (Grant Instruments, Cambridge, UK) for $30 \mathrm{~min}$ at $4^{\circ} \mathrm{C}$. In general, Dynabeads have the capacity to isolate $15 \mu \mathrm{g}$ EDIII/mg beads. Naked Dynabeads served as a negative control and were incubated with binding buffer only under the same conditions. After washing thoroughly as mentioned above, the serum sample was incubated with the Dynabead-EDIII complex on a roller for $2 \mathrm{~h}$ at $4^{\circ} \mathrm{C}$. This depletion procedure for each serum sample was repeated at least three times with a new Dynabead-EDIII complex, and the depletion efficiency was confirmed by a double-antigen sandwich ELISA with rEDIII. 
In vitro neutralization assay using enzyme-linked immunospot microneutralization test (ELISPOT-MNT). The ELISPOT-MNT was performed as previously described with several modifications (28). Briefly, serially diluted serum samples were blended with equal volumes of virus containing 200 PFUs per well, then serum and virus mixtures were incubated for $1 \mathrm{~h}$ at $37^{\circ} \mathrm{C}$. The serum and virus mixtures were then added to the LLC-MK2 cell monolayers cultured in 96-well plates (Greiner Bio-One, Frickenhausen, Germany) and incubated for $1.5 \mathrm{~h}$. Next, these cells were covered with semi-solid MEM medium containing 1\% methylcellulose (Sigma-Aldrich) and $5 \%$ heat-inactivated FBS. The cell monolayers were incubated at $37^{\circ} \mathrm{C}$ for two to three days. Then the cells were fixed and stained with the anti-DENV non-structural protein 1 (NS1) monoclonal antibody 5F10A7. Following the addition of HRP-conjugated polyclonal goat anti-mouse IgG (1:300; cat no. A5278; Sigma-Aldrich), plaques were visualized with AEC solution (SK4200; Vector Laboratories, Burlingame, CA, USA) and counted with an automated ELISPOT instrument (Cellular Technology Ltd., Shaker Heights, OH, USA). A $50 \%$ reduction in the number of plaques compared with the virus dose control absenting a target serum was calculated as follows: $\%$ reduction $=100 \times[1$-(average number of plaques for each dilution/average number of plaques for virus dose control)].

In vitro enhancement assay by NS1 capture ELISA-based ADE assay (ELISA-ADE). The assessment of ADE by measuring DENV NS1 production in Fc $\gamma$ receptor-expressing K562 cell culture supernatants was performed by modified ELISA-ADE as previously described (29). Briefly, serially diluted serum samples were incubated with an equal volume of DENV at a multiplicity of infection of 0.5 or 0.125 in 96 -well plates for $1 \mathrm{~h}$ at $37^{\circ} \mathrm{C}$. The $\mathrm{K} 562$ cells were adjusted to a concentration of $5 \times 10^{4} / 100 \mu \mathrm{l}$ and added to the virus-antibody mixture. Following incubation for $2 \mathrm{~h}$ at $37^{\circ} \mathrm{C}$, the K562 cells were washed twice and cultured in RPMI-1640 medium containing $5 \%$ FBS for 4 days at $37^{\circ} \mathrm{C}$. The 96 -well plates were placed at $-80^{\circ} \mathrm{C}$ overnight, and the NS1 levels in the freeze-thawed 96-well plate supernatants were used to evaluate the severity of viral infection using an NS1 antigen-capture ELISA previously developed in our laboratory (23). To distinguish between enhancing and non-enhancing activities, a cut-off value was set as a mean absorbance at $450 \mathrm{~nm}$ plus 3 standard deviations using eight wells containing the virus control in the absence of antibodies. The K562 cells incubated with DENV alone were set as the infection baseline from which fold enhancement was determined.

Statistical analysis. Statistical analyses were performed using GraphPad Prism version 5.04 (GraphPad Software Inc., La Jolla, CA, USA) and Predictive Analytics Suite Workstation (PASW; version 20.0; IBM SPSS, Armonk, NY, USA). The $50 \%$ neutralizing titers (NT50) of the sera corresponding to a $50 \%$ plaque reduction, were computed using a nonlinear regression (curve fit) analysis. The peak enhancement titre (PET) of the serum corresponded to the serum dilution in the highest fold enhancement. The differences in neutralization among the four DENV serotypes in the human convalescent serum samples were compared by the analysis of variance

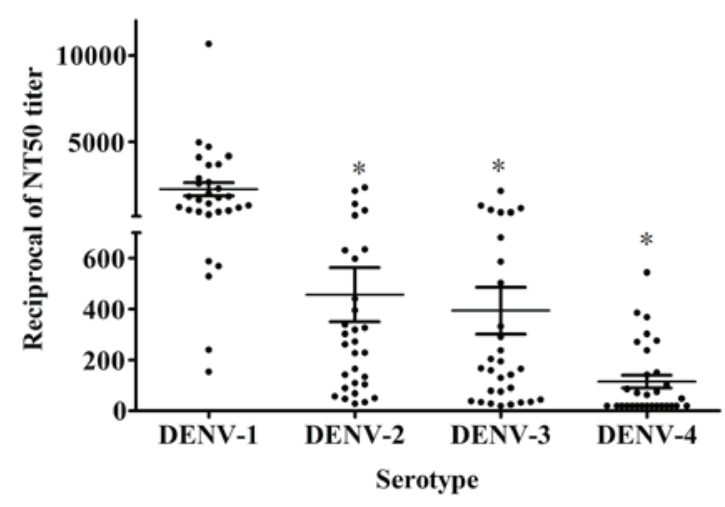

Figure 1. Neutralization titres against all four DENV serotypes in convalescent sera from DENV-1-infected patients. The neutralization titres of thirty serum samples were measured using enzyme-linked immunospot microneutralization test. The NT50 neutralization titres of the serum samples were expressed as reciprocal serum dilutions at which the viral infection was inhibited by $50 \%$. * $\mathrm{P}<0.05$ vs. DENV-1. DENV, dengue virus; NT50, 50\% neutralizing titers.

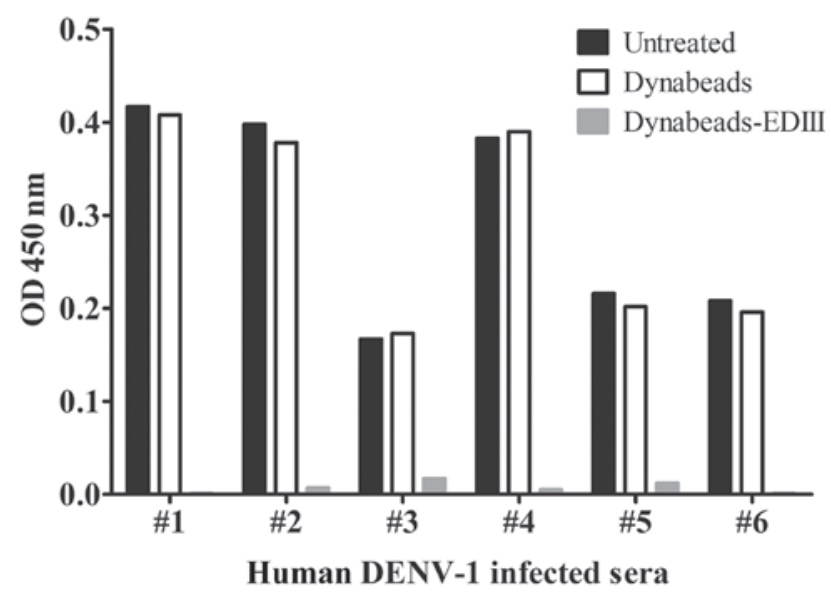

Figure 2. Binding properties of EDIII-reactive antibodies in DENV-1-infected human sera. Six primary DENV-1-infected convalescent human serum samples \#1-6 were depleted of homologous EDIII-reactive antibodies using Dynabead-EDIII conjugates. Total homologous EDIII-reactive antibodies were assessed for DENV-1 EDIII binding by double-antigen sandwich enzyme-linked immunosorbent assay, and the absolute OD value at $450 \mathrm{~nm}$ is shown on the y-axis. The data display one of two representative experiments. EDIII, envelope domain III; DENV, dengue virus; OD, optical density.

and least significant difference multiple comparison tests. The correlation between neutralization activities and EDIII-specific antibody titres to DENV-1 in the human convalescent serum samples was calculated by Spearman's rank correlation test. The neutralization and enhancement differences of the six DENV-1-infected sera prior to and following EDIII antibody-depletion were compared with analysis of variance.

\section{Results}

Correlation between neutralization and EDIII-reactive antibody titres in convalescent sera from DENV-1-infected patients. To evaluate the contribution of EDIII-reactive antibodies to the total neutralization activity of convalescent sera from patients infected with DENV-1, serum titres 
DENV-1

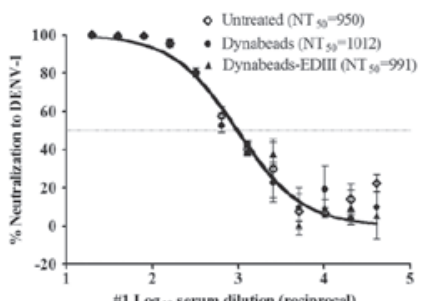

- Untreated (NT $s_{00}=1190$
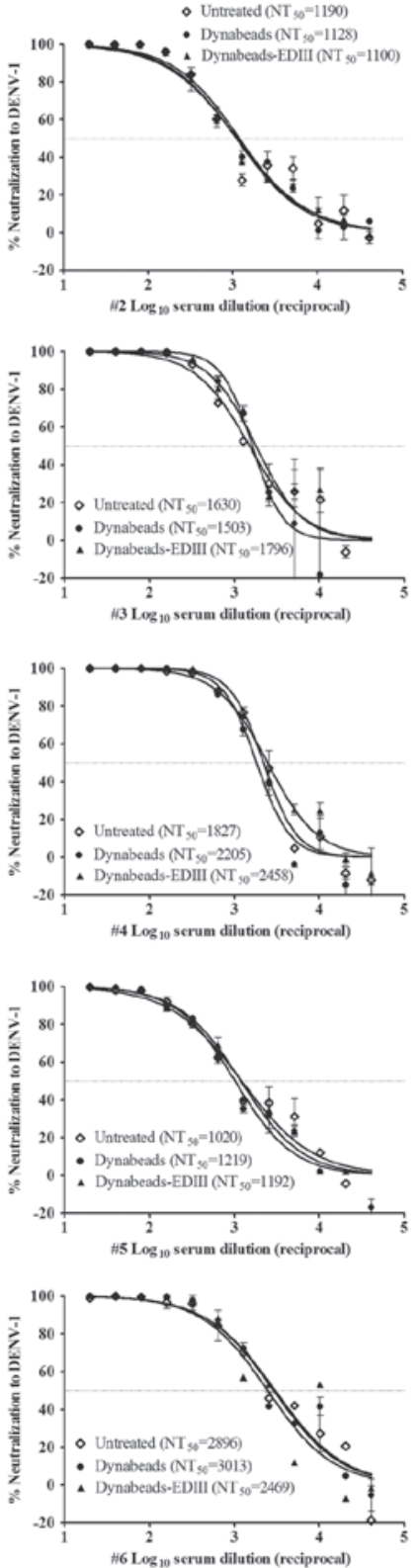

DENV-2
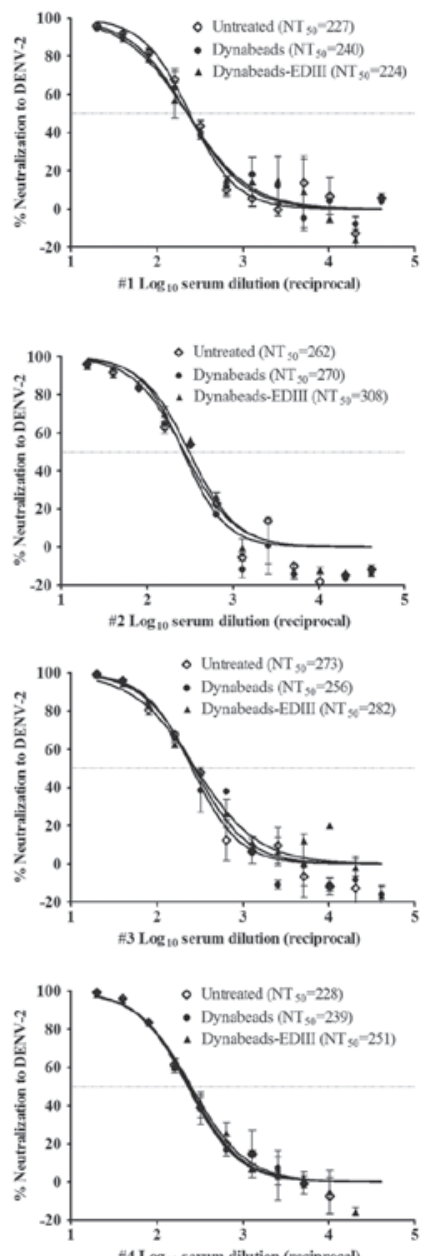

fit $\mathrm{L}^{2} \mathrm{~g}_{10}$ serum dilution (reciprocal)
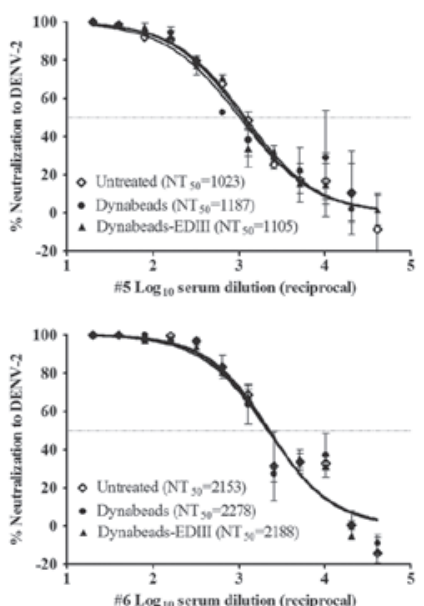

DENV-3
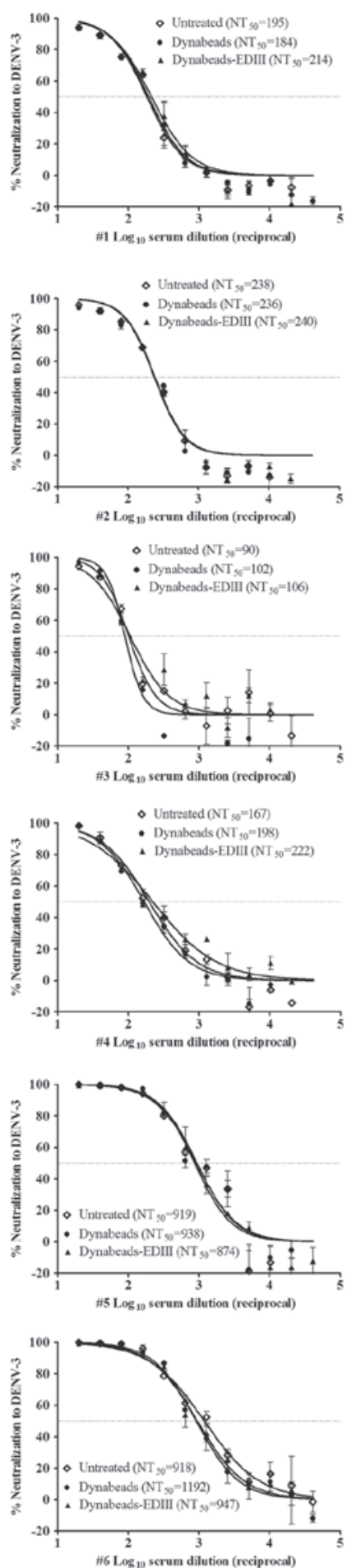

DENV-4
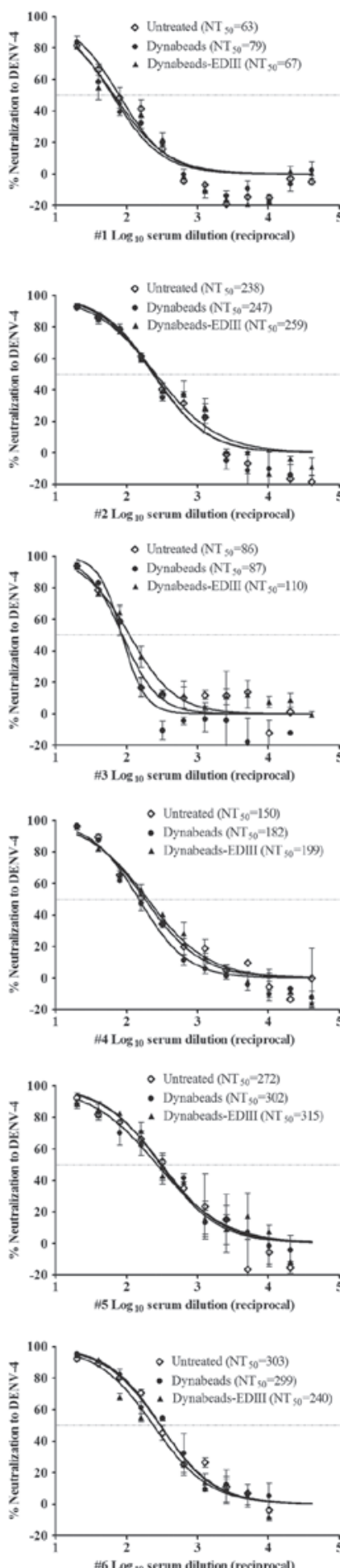

Figure 3. Neutralization properties of EDIII-reactive antibodies in DENV-1-infected human sera. The neutralization activities of the above six DENV-1-infected human serum samples for all four DENV serotypes were measured in parallel in untreated, Dynabeads and Dynabead-DENV-1 EDIII absorption serum samples. The percentage of plaque reduction is shown on the $y$-axis, the log reciprocal dilution of the serum is shown on the $\mathrm{x}$-axis, and the dashed line indicates $50 \%$ plaque reduction. For each serum sample, the neutralization curve fits generated by nonlinear regression analysis are presented. The data show one of two independent experiments in duplicate. The error bars represent the standard error of the mean of duplicate wells. EDIII, envelope domain III; DENV, dengue virus; NT50, 50\% neutralizing titers.

specific to DENV-1 recombinant EDIII (rEDIII) protein in 30 human serum samples were detected by a double-antigen sandwich ELISA. In addition, the NT50 of these sera were detected by ELISPOT-MNT. The results indicated that the
EDIII-binding antibody titres of the patient serum samples were very low that was not correlated with the serum NT50 titres $(\mathrm{R}=0.215, \mathrm{P}=0.253)$ (Table I), implying that EDIII-reactive antibodies may not exhibit a major role in 

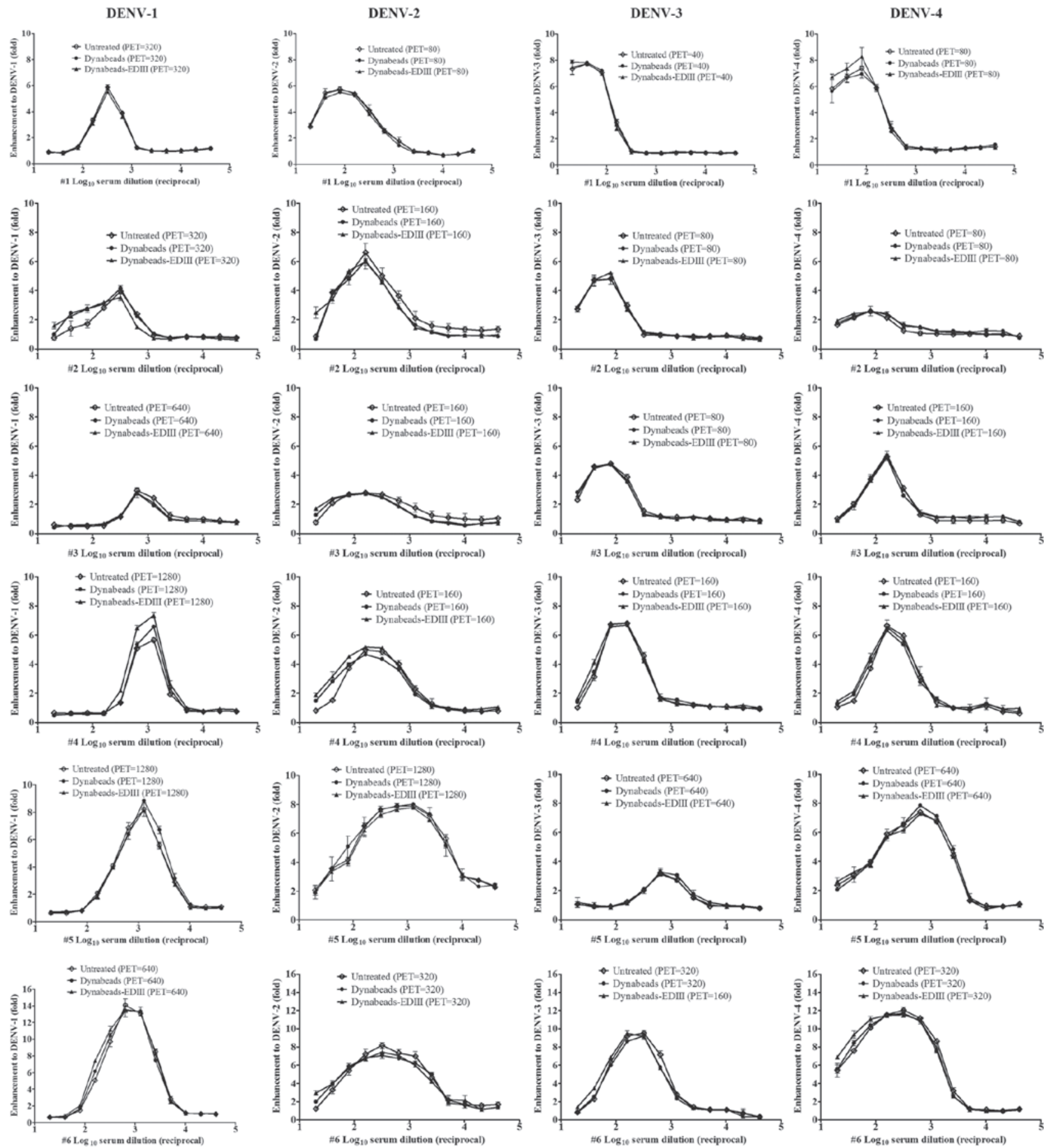

Figure 4. Enhancement properties of EDIII-reactive antibodies in DENV-1-infected human sera. The peak enhancement titres of thirty serum samples were assessed using ELISA-ADE. The ADE activities of the six DENV-1-infected human serum samples for all four dengue serotypes were measured in untreated, Dynabead and Dynabead-DENV-1 recombinant EDIII absorption serum samples. Fold enhancement is shown on the y-axis, and the log reciprocal dilution of the serum is shown on the x-axis. The data show one of two independent experiments in duplicate. The error bars represent the standard error of the mean of duplicate wells. EDIII, envelope domain III; DENV, dengue virus; NT50, 50\% neutralizing titers; PET, peak enhancement titre; ADE, antibody-dependent enhancement.

neutralization in DENV-infected human sera. Furthermore, the mean reciprocal NT50 values for DENV-1, DENV-2, DENV-3 and DENV-4 in these 30 sera were 2250, 455.8, 393.6 and 114.1, respectively. A significant difference was identified among four serotypes of NT50 in these 30 sera $(\mathrm{F}=15.412, \mathrm{P}<0.001)$ and the sera had a significantly higher NT50 to DENV-1 than the heterogeneous serotypes $(\mathrm{P}<0.05)$
(Fig. 1 and Table I), which are consistent with the DENV-1 infection profile.

Role of EDIII-reactive antibodies in human DENV-1-infected sera. To further identify the functional properties of EDIII-reactive antibodies in DENV-1 convalescent serum, the neutralization and ADE activities of EDIII antibody-depleted 
A

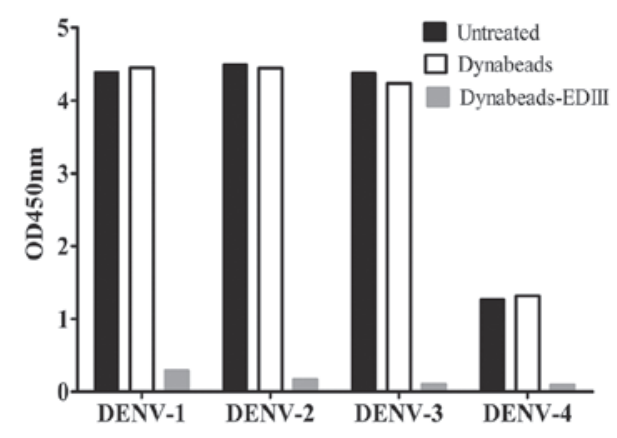

B
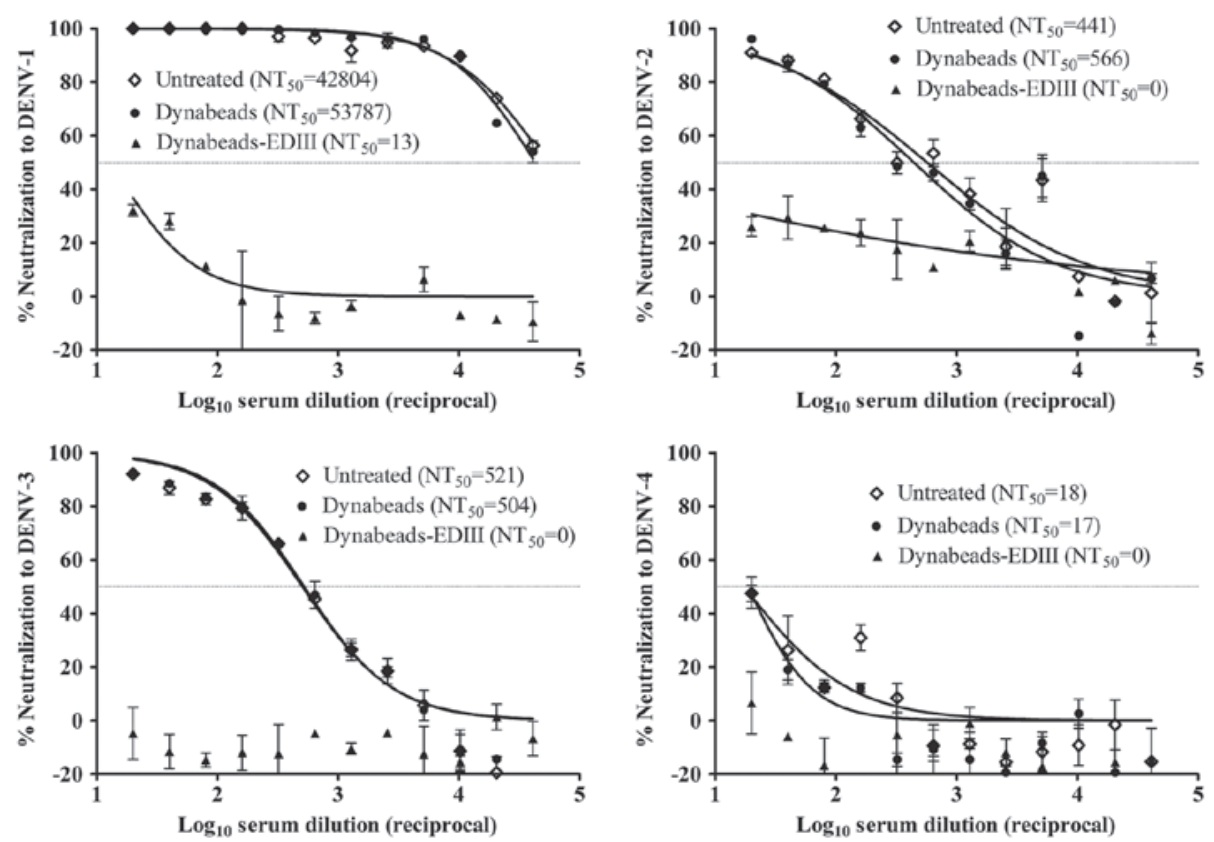

C
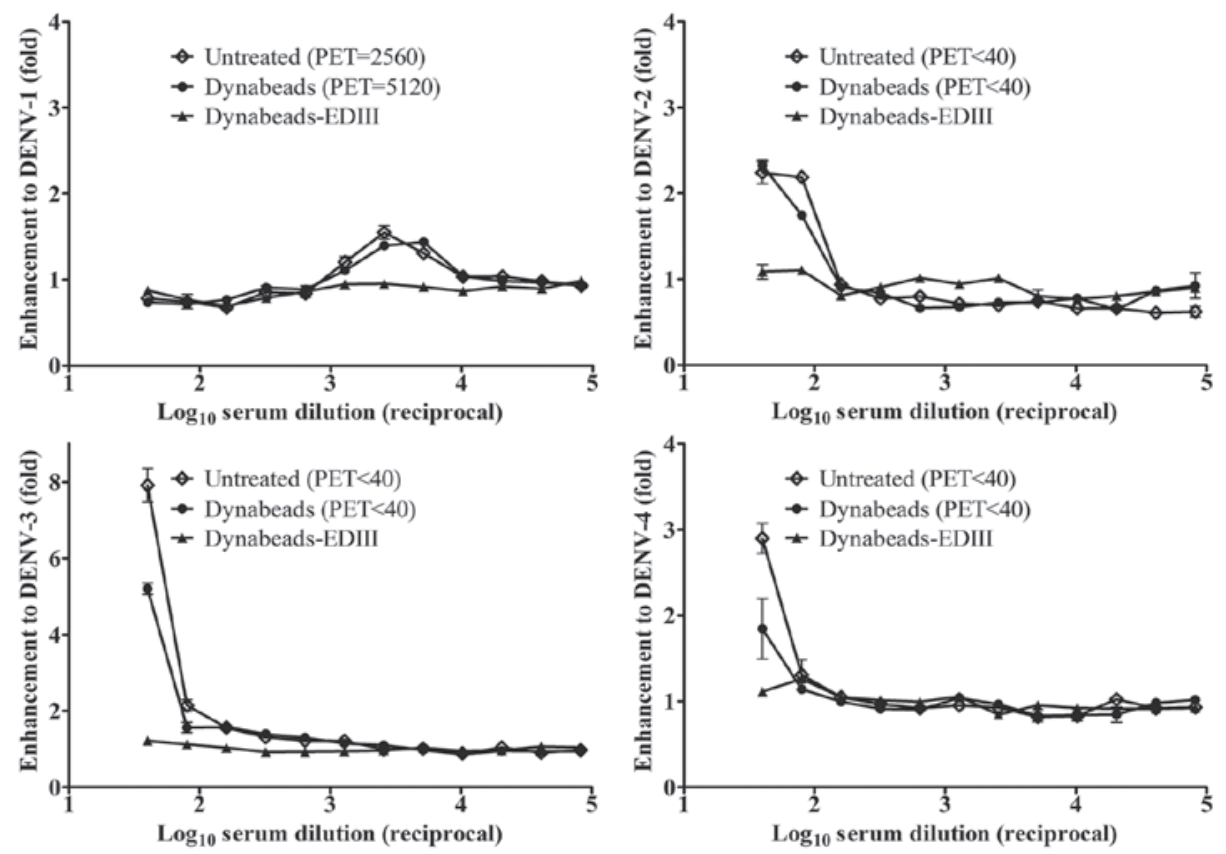

Figure 5. Functional properties of EDIII-reactive antibodies in rabbit antiserum. (A) EDIII-reactive antibodies were depleted from rabbit antiserum using Dynabead-DENV-1 reEDIII, and the antiserum was assessed for DENV-1, DENV-2, DENV-3 and DENV-4 EDIII-binding properties by ELISA. The neutralization (B) and enhancement (C) properties for the four dengue serotypes in the rabbit antiserum before and after the depletion of EDIII-reactive antibodies were measured with enzyme-linked immunospot microneutralization test and ELISA-ADE. The data display one of two representative experiments. The error bars represented the standard error of the mean of duplicate wells. EDIII, envelope domain III; DENV, dengue virus; NT50, 50\% neutralizing titers; PET, peak enhancement titre; ADE, antibody-dependent enhancement; ELISA, enzyme-linked immunosorbent assay. 
sera were measured. First, six human convalescent serum samples from patients with primary DENV-1 infection were incubated with Dynabeads-DENV-1 rEDIII conjugates to deplete the EDIII-binding antibodies. As shown in Fig. 2, the depletion successfully removed the EDIII-reactive antibodies in the sera compared with the naked Dynabead absorption control. Then, ELISPOT-MNT and ELISA-ADE were performed to measure the neutralization and ADE activities of the EDIII-specific antibody-depleted sera. Untreated sera and Dynabead absorption sera were used as controls. No significant in the neutralization titers in the group treated with Dynabeads-EDIII compared with the group treated with Dynabeads control in the six sera samples for DENV-1 $(\mathrm{F}=0.030, \mathrm{P}=0.971), \mathrm{DENV}-2(\mathrm{~F}=0.006, \mathrm{P}=0.994), \mathrm{DENV}-3$ $(\mathrm{F}=0.028, \mathrm{P}=0.972)$ and DENV-4 $(\mathrm{F}=0.039, \mathrm{P}=0.962)$ (Fig. 3$)$. In addition, there was no observed significant difference in the enhancement in the group treated with Dynabeads-EDIII compared with the group treated with Dynabeads control in the 6 sera samples for DENV-1 $(\mathrm{F}=0.000 ; \mathrm{P}>0.05$, DENV-2 $(\mathrm{F}=0.000 ; \mathrm{P}>0.05), \mathrm{DENV}-3(\mathrm{~F}=0.028 ; \mathrm{P}=0.973)$ and DENV-4 ( $\mathrm{F}=0.000 ; \mathrm{P}>0.05)$ (Fig. 4). The results indicate no significant loss of neutralization or ADE activity for the four dengue serotypes after the removal of EDIII-specific antibodies from the human sera. Therefore, this suggested that EDIII-specific antibodies in primary DENV-1-infected convalescent sera exhibit minor roles in the neutralization and enhancement of viral replication.

Role of EDIII-reactive antibodies in rabbit antiserum. Previously, a patient was shown to develop serum antibodies against various DENV proteins after DENV infection (30-33). Based on the consideration that EDIII may be a non-predominant antigen in complex antigenic components of DENV, and EDIII could not induce effective antibody response in natural infection because of the antigenic competition, the present study aimed to determine whether purified DENV-1 rEDIII protein would result in effective protective neutralizing antibody, in addition to the roles of EDIII reactive antibodies in rabbit antiserum. The rabbit antiserum exhibited a strong binding capacity to recombinant EDIII protein with a titre of 1:10,000, which was verified by double antigen sandwich ELISA as that used for human convalescent sera (data not shown), and further confirmed by removing EDIII-specific antibodies using Dynabead-DENV-1 rEDIII (Fig. 5A). Notably, the antiserum displayed concentration-dependent neutralization, in which the rabbit antiserum exhibited an NT50 of $\sim 50,000$ for DENV-1, and an NT50 of $~ 500$ for DENV-2 and DENV-3, but without neutralization of DENV-4 (Fig. 5B). In contrast to its neutralization activity, the rabbit antiserum showed markedly lower dilution-dependent ADE activity, and the PET against DENV-1 $(\sim 1: 5,000)$ was higher than that of the other serotypes $(<1: 40)$ (Fig. 5C). All of the above neutralization and ADE activities were mostly depleted by absorption with Dynabead-EDIII conjugates (Fig. 5B and C). These results indicated that antibodies induced by DENV-1 rEDIII protein in rabbit serum possess stronger neutralization activity and lower ADE on homotypic virus replication, and the lower neutralization and ADE on heterotypic infection. The results also suggest that only a small fraction of rabbit anti-EDIII antibodies could enhance an infection with heterotypic DENV.

\section{Discussion}

In this study, the correlation between serum neutralization titres and EDIII-reactive antibody titres binding to EDIII protein was determined in 30 convalescent serum samples from patients with primary DENV-1 infection as shown in Table I. Notably, no correlation was identified between high levels of serum NT50 titres and low levels of EDIII-binding titres in patient serum samples implies that EDIII-reactive antibodies may not be involved in neutralization. In total, 6 of 30 convalescent serum samples were treated with Dynabead-DENV-1 rEDIII to remove specific antibodies and it was demonstrated that the serum neutralization and enhancement activities on homotypic and heterotypic DENV replication were unchanged prior to and following the depletion. The depletion with Dynabead-EDIII was demonstrated to be sufficient and specific, since the recombinant EDIII proteins were expressed with the methylotrophic yeast, Pichia pastoris, which is closer to the conformation of natural EDIII than the prokaryotic expression protein conjugates (34-36). This depletion further confirmed that EDIII-specific antibodies may be insignificant for the antibody protective response in human convalescent sera, which is in accordance with the reports on DENV by several groups $(11,20-22)$.

To identify the role of EDIII-reactive antibodies by effective immunization, recombinant EDIII protein was purified for immunization to prepare rabbit antiserum. In addition to a high titre of binding to DENV1 rEDIII protein, the EDIII-reactive antibodies of rabbit antiserum showed strong neutralization activity and corresponding ADE activity on homologous serotype virus DENV-1, compared with the weak neutralization and ADE on heterogeneous virus infection. All the above-mentioned activities of rabbit antiserum were completely removed by treatment with Dynabead-EDIII. These results suggest that EDIII-reactive antibodies are critical in the neutralization and ADE activities in rabbit antiserum. Notably, the results also suggest that the ADE of heterotypic DENV only occurs at high concentrations of anti-EDIII antibody, which means that only a small fraction of the anti-EDIII antibody in rabbit antiserum possess ADE activity.

These results supported our hypothesis that DENV EDIII may be not a predominant antigen in complex components of DENV under antigen competition in vivo, consequently, DENV EDIII could not induce an effective antibody neutralization or ADE response in the natural infection process. However, immunization using purified recombinant EDIII protein without antigen competition by other virus components could provoke the antibody response with strong neutralization and weak ADE effects, which suggests that this protein could be considered as a subunit vaccine candidate. There were also reports that EDIII-specific human antibody can inhibit DENV infection in a dose-dependent manner (37), a tetravalent vaccine containing two bivalent DENV EDIIIs could induce protective antibody responses against all four DENV serotypes (38), and $>50 \%$ of the total $\mathrm{IgG}$ was targeted against EDIII when mice were vaccinated with DENV virus-like particles (39). In our previous study, two monoclonal antibodies were generated, 
2D73 and 3E31, and were shown to possess strong neutralization abilities, but only 2D73 caused ADE (data not shown). This finding may be attributed to the structure of the different epitopes of EDIII protein, which may affect the properties of antibody response, particularly the production of neutralizing or enhancing antibodies. It has been demonstrated that an improvement in the anti-DENV immune response with increasing neutralizing may be achieved by modifying the DENV EDIII protein $(40,41)$. Thus, we hypothesise that the ideal EDIII epitope candidate or epitope combinations would be identified or restructured to induce the antibody response with strong neutralization and weak or no ADE.

The ideal dengue vaccine should induce a life-long protective response against all four DENV serotypes, thereby preventing the risk of ADE. Thus far, only a chimeric recombinant DNA dengue vaccine developed by Sanofi Pasteur completed clinical trial phase 3 , this vaccine can provide $60 \%$ protection to DENV-1, and $80-90 \%$ protection against DENV-3 and DENV-4, but no protection against DENV-2 (42). Based on the epidemiological and aetiological characteristics of dengue infection in southern China, DENV-1 is the most predominant serotype (accounting for $>60 \%$ of all patients), DENV-2 is occasionally detected, and DENV-3 and DENV-4 are seldom observed (43-46). Therefore, our research has been focused primarily on DENV-1 EDIII in human convalescent serum samples and DENV-1 EDIII immunized rabbit serum, in which the neutralization and ADE on heterotypic DENVs were also observed. In conclusion, the present study demonstrated that purified DENV-1 EDIII protein as a vaccine candidate would be safe to protect from heterotypic DENV infections.

\section{Acknowledgements}

This study was supported by grants from the National Science Foundation for Young Scientists of China (grant no. 81201296 to Mr. Kun Wen), the NSFC-Guangdong Joint Fund (grant no. U1132002 to Professor Xiao-yan Che) and 2009 and the project was also supported by the Guangdong Province Universities and Colleges Pearl River Scholar Funded Scheme.

\section{References}

1. Gutiérrez G, Gresh L, Pérez MÁ, Elizondo D, Avilés W, Kuan G, Balmaseda A and Harris E: Evaluation of the diagnostic utility of the traditional and revised WHO dengue case definitions. PLoS Negl Trop Dis 7: e2385, 2013.

2. Horstick O, Jaenisch T, Martinez E, Kroeger A, See LL, Farrar J and Ranzinger SR: Comparing the usefulness of the 1997 and 2009 WHO dengue case classification: A systematic literature review. Am J Trop Med Hyg 91: 621-634, 2014.

3. Narvaez F, Gutierrez G, Pérez MA, Elizondo D, Nuñez A, Balmaseda A and Harris E: Evaluation of the traditional and revised WHO classifications of Dengue disease severity. PLoS Negl Trop Dis 5: e1397, 2011.

4. Henchal EA and Putnak JR: The dengue viruses. Clin Microbiol Rev 3: 376-396, 1990

5. Sabin AB: Research on dengue during World War II. Am J Trop Med Hyg 1: 30-50, 1952.

6. Rothman AL: Dengue: Defining protective versus pathologic immunity. J Clin Invest 113: 946-951, 2004.

7. Halstead SB: Neutralization and antibody-dependent enhancement of dengue viruses. Adv Virus Res 60: 421-467, 2003.
8. Kuhn RJ, Zhang W, Rossmann MG, Pletnev SV, Corver J, Lenches E, Jones CT, Mukhopadhyay S, Chipman PR, Strauss EG, et al: Structure of dengue virus: Implications for flavivirus organization, maturation, and fusion. Cell 108: 717-725, 2002.

9. Rey FA, Heinz FX, Mandl C, Kunz C and Harrison SC: The envelope glycoprotein from tick-borne encephalitis virus at $2 \mathrm{~A}$ resolution. Nature 375: 291-298, 1995.

10. Crill WD, Hughes HR, Delorey MJ and Chang GJ: Humoral immune responses of dengue fever patients using epitope-specific serotype-2 virus-like particle antigens. PLoS One 4: e4991, 2009.

11. Wahala WM, Kraus AA, Haymore LB, Accavitti-Loper MA and de Silva AM: Dengue virus neutralization by human immune sera: Role of envelope protein domain III-reactive antibody. Virology 392: 103-113, 2009.

12. Crill WD and Roehrig JT: Monoclonal antibodies that bind to domain III of dengue virus E glycoprotein are the most efficient blockers of virus adsorption to Vero cells. J Virol 75: 7769-7773, 2001.

13. Chua AJ, Vituret C, Tan ML, Gonzalez G, Boulanger P, Ng ML and Hong SS: A novel platform for virus-like particle-display of flaviviral envelope domain III: Induction of Dengue and West Nile virus neutralizing antibodies. Virol J 10: 129, 2013

14. Chen HW, Liu SJ, Li YS, Liu HH, Tsai JP, Chiang CY, Chen MY, Hwang CS, Huang CC, Hu HM, et al: A consensus envelope protein domain III can induce neutralizing antibody responses against serotype 2 of dengue virus in non-human primates. Arch Virol 158: 1523-1531,2013.

15. Lok SM, Kostyuchenko V, Nybakken GE, Holdaway HA, Battisti AJ, Sukupolvi-Petty S, Sedlak D, Fremont DH, Chipman PR, Roehrig JT, et al: Binding of a neutralizing antibody to dengue virus alters the arrangement of surface glycoproteins. Nat Struct Mol Biol 15: 312-317, 2008.

16. Matsui K, Gromowski GD, Li L and Barrett AD: Characterization of a dengue type-specific epitope on dengue 3 virus envelope protein domain III. J Gen Virol 91: 2249-2253, 2010.

17. Rajamanonmani R, Nkenfou C, Clancy P, Yau YH, Shochat SG, Sukupolvi-Petty S, Schul W, Diamond MS, Vasudevan SG and Lescar J: On a mouse monoclonal antibody that neutralizes all four dengue virus serotypes. J Gen Virol 90: 799-809, 2009.

18. Wahala WM, Donaldson EF, de Alwis R, Accavitti-Loper MA, Baric RS and de Silva AM: Natural strain variation and antibody neutralization of dengue serotype 3 viruses. PLoS Pathog 6: e1000821, 2010.

19. Yamanaka A, Kosugi S and Konishi E: Infection-enhancing and -neutralizing activities of mouse monoclonal antibodies against dengue type 2 and 4 viruses are controlled by complement levels. J Virol 82: 927-937, 2008

20. de Alwis R, Smith SA, Olivarez NP, Messer WB, Huynh JP, Wahala WM, White LJ, Diamond MS, Baric RS, Crowe JE Jr and de Silva AM: Identification of human neutralizing antibodies that bind to complex epitopes on dengue virions. Proc Natl Acad Sci USA 109: 7439-7444, 2012.

21. Midgley CM, Bajwa-Joseph M, Vasanawathana S, Limpitikul W, Wills B, Flanagan A, Waiyaiya E, Tran HB, Cowper AE, Chotiyarnwong $\mathrm{P}$, et al: An in-depth analysis of original antigenic sin in dengue virus infection. J Virol 85: 410-421, 2011.

22. Williams KL, Wahala WM, Orozco S, de Silva AM and Harris E: Antibodies targeting dengue virus envelope domain III are not required for serotype-specific protection or prevention of enhancement in vivo. Virology 429: 12-20, 2012.

23. Ding X, Hu D, Chen Y, Di B, Jin J, Pan Y, Qiu L, Wang Y, Wen K, Wang M and Che X: Full serotype- and group-specific NS1 capture enzyme-linked immunosorbent assay for rapid differential diagnosis of dengue virus infection. Clin Vaccine Immunol 18: 430-434, 2011.

24. Cai JP, Qian F, Wang JY, Zhao Y, Xu XJ, Jin WR and Che XY: Characterization and secreted expression of dengue virus type I-IV envelope glycoprotein domain III in Pichia pastoris. Zhonghua Yu Fang Yi Xue Za Zhi 44: 721-725, 2010 (In Chinese).

25. Knapp W, Holubar K and Wick G: Immunofluorescence and related staining techniques. Journal 215, 1978.

26. Bai Z, Liu L, Tu Z, Yao L, Liu J, Xu B, Tang B, Liu J, Wan Y, Fang $\mathrm{M}$, et al: Real-time PCR for detecting circulating dengue virus in the Guangdong Province of China in 2006. J Med Microbiol 57: 1547-1552, 2008.

27. Bai Z, Liu L, Tu Z, Yao L, Liu J, Xu B, Tang B, Liu J, Wan Y, Fang M, et al: Real-time PCR for detecting circulating dengue virus in the Guangdong Province of China in 2006. J Med Microbiol 57: 1547-1552, 2008. 
28. Liu L, Wen K, Li J, Hu D, Huang Y, Qiu L, Cai J and Che X: Comparison of plaque- and enzyme-linked immunospot-based assays to measure the neutralizing activities of monoclonal antibodies specific to domain III of dengue virus envelope protein. Clin Vaccine Immunol 19: 73-78, 2012.

29. Li XQ, Chen J, Huang YF, Ding XX, Liu LD, Qiu LW, Pan YX, Deng YQ, Hu DM, Di B, et al: Evaluation and analysis of dengue virus enhancing and neutralizing activities using simple high-throughput assays. Appl Microbiol Biotechnol 97: 6503-6511, 2013.

30. Wahala WM and Silva AM: The human antibody response to dengue virus infection. Viruses 3: 2374-2395, 2011.

31. Valdés K, Alvarez M, Pupo M, Vázquez S, Rodriguez R and Guzmán MG: Human dengue antibodies against structural and nonstructural proteins. Clin Diagn Lab Immunol 7: 856-857, 2000.

32. Lai CY, Tsai WY, Lin SR, Kao CL, Hu HP, King CC, Wu HC, Chang GJ and Wang WK: Antibodies to envelope glycoprotein of dengue virus during the natural course of infection are predominantly cross-reactive and recognize epitopes containing highly conserved residues at the fusion loop of domain II. J Virol 82: 6631-6643, 2008.

33. Abubakar S, Azila A, Suzana M and Chang LY: Antigenic cell associated dengue 2 virus proteins detected in vitro using dengue fever patients sera. Malays J Pathol 24: 29-36, 2002.

34. Cregg JM, Vedvick TS and Raschke WC: Recent advances in the expression of foreign genes in Pichia pastoris. Biotechnology (NY) 11: 905-910, 1993.

35. Higgins DR: Overview of protein expression in Pichia pastoris. Curr Protoc Protein Sci Chapter 5: Unit5 7, 2001.

36. Ramón A and Marín M: Advances in the production of membrane proteins in Pichia pastoris. Biotechnol J 6: 700-706, 2011.

37. Saokaew N, Poungpair O, Panya A, Tarasuk M, Sawasdee N, Limjindaporn T, Chaicumpa W and Yenchitsomanus P: Human monoclonal single-chain antibodies specific to dengue virus envelope protein. Lett Appl Microbiol 58: 270-277, 2014.
38. Zhao H, Jiang T, Zhou XZ, Deng YQ, Li XF, Chen SP, Zhu SY, Zhou X, Qin ED and Qin CF: Induction of neutralizing antibodies against four serotypes of dengue viruses by MixBiEDIII, a tetravalent dengue vaccine. PLoS One 9: e86573, 2014.

39. Galula JU, Shen WF, Chuang ST, Chang GJ and Chao DY: Virus-like particle secretion and genotype-dependent immunogenicity of dengue virus serotype 2 DNA vaccine. J Virol 88: 10813-10830, 2014.

40. Chiang CY, Pan CH, Hsieh CH, Tsai JP, Chen MY, Liu HH, Liu SJ, Chong P, Leng CH and Chen HW: Lipidated dengue-2 envelope protein domain III independently stimulates long-lasting neutralizing antibodies and reduces the risk of antibody-dependent enhancement. PLoS Negl Trop Dis 7: e2432, 2013.

41. Coconi-Linares N, Ortega-Dávila E, López-González M, García-Machorro J, García-Cordero J, Steinman RM, Cedillo-Barrón L and Gómez-Lim MA: Targeting of envelope domain III protein of DENV type 2 to DEC-205 receptor elicits neutralizing antibodies in mice. Vaccine 31: 2366-2371, 2013.

42. Sinha G: Sanofi's dengue vaccine first to complete phase 3. Nat Biotechnol 32: 605-606, 2014.

43. Wu W, Bai Z, Zhou H, Tu Z, Fang M, Tang B, Liu J, Liu L, Liu J and Chen W: Molecular epidemiology of dengue viruses in southern China from 1978 to 2006. Virol J 8: 322, 2011.

44. Jiang L, Wu X, Wu Y, Bai Z, Jing Q, Luo L, Dong Z, Yang Z, $\mathrm{Xu}$ Y, Cao Y, et al: Molecular epidemiological and virological study of dengue virus infections in Guangzhou, China, during 2001-2010. Virol J 10: 4, 2013.

45. Guo RN, Lin JY, Li LH, Ke CW, He JF, Zhong HJ, Zhou HQ, Peng ZQ, Yang F and Liang WJ: The prevalence and endemic nature of dengue infections in Guangdong, South China: An epidemiological, serological, and etiological study from 2005-2011. PLoS One 9: e85596, 2014.

46. Guo R, Peng Z, Song T, He J, Zhong H, Li L and Liang W: Current infection status and epidemic risk analysis of dengue fever and Chikungunya in Guangdong province, from 1990 to 2012. Zhonghua Liu Xing Bing Xue Za Zhi 35: 167-169, 2014 (In Chinese). 